\title{
Computer-aided craniofacial superimposition using a quasi-Newton iterative closest point approach
}

\author{
Joi San Tan ${ }^{\mathrm{a}, *}$, Ibrahim Venkat ${ }^{\mathrm{a}}$, Paul T. Jayaprakash ${ }^{\mathrm{b}}$ \\ a School of Computer Sciences, Universiti Sains Malaysia, 11800 USM, Penang, Malaysia \\ b School of Health Sciences, Health Campus, Universiti Sains Malaysia, 16150 Kubang Kerian, Kelantan, \\ Malaysia
}

*Corresponding author, e-mail: hiki_3joi@yahoo.com

Received 17 Nov 2014

Accepted 19 Feb 2016

\begin{abstract}
Craniofacial superimposition is a forensic imaging technique used to identify an unknown skull by matching it with the available face photographs of missing individuals. Life-size enlargement of the face image and orientating the skull to correspond to the posture seen in the face photograph are the two main problems that exist in conventional as well as in the computer-aided craniofacial superimposition. Here we address these two potential issues by proposing a novel computer-aided approach which uses the quasi-Newton optimization method and iterative closest point algorithm. The results showed that the quasi-Newton method proposed is able to eliminate $76 \%$ and $66 \%$ of false matches, respectively, for the male and the female skull superimpositions during the initial stage of filtration. Our experimental results demonstrate that the proposed approach is efficient in assigning face photographs as inclusions (positives) and exclusions (negatives) while superimposing with related and unrelated skulls.
\end{abstract}

KEYWORDS: forensic science, Whitnall's tubercle

\section{INTRODUCTION}

When DNA-based identification becomes impossible or is not reliable, skull-photo superimposition is an alternative since face photographs are commonly available. Such situations include the discovery of skeletonized remains or dead bodies in advanced state of decomposition. Craniofacial superimposition (CS) is carried out to establish probable identity of a skull by comparing it with the given face photograph.

Computer-aided superimposition (CAS) can be divided into two types. The first type is a set of computer assisted processes that use commercial software such as PHOTOSHOP, CORAL DRAW or 3D STUDio MAX to complete the skull-face overlay manually in digital form ${ }^{1,2}$. The second type is a set of computational intelligence techniques such as genetic algorithms $(\mathrm{GA})^{3-5}$, multimodal $\mathrm{GA}^{6}$, covariance matrix adaptation evolution strategy ${ }^{7}$, cooperative co-evolutionary approach ${ }^{8}$, and realcoded $\mathrm{GA}^{9,10}$ that are used to automate the overlay process. These intelligent techniques aid to mitigate the uncertainty elements such as fuzzy landmarks and thickness of soft tissues that are encountered during the process of mapping the 3D-skull model onto the $2 \mathrm{D}$ face. The mapping process in turn deploys affine and perspective transformations (rotation, scaling, translation, angle of rotation, and perspective angle of view) as part of the CAS approach.

Two parameters that have been stressed in skullphoto superimposition are the use of life size images and anatomically appropriate orientation of the skull ${ }^{11-13}$. The CAS approaches discussed are found to have two common problems: failure to achieve appropriate orientation of the skull and lifesize magnification of the images. The first problem is due to the sole reliance on the landmarks in the frontal plane of the face image ${ }^{1-3,14}$ for orienting the skull. Santamaría et $\mathrm{al}^{15}$ discussed the coplanarity problem in forensic craniofacial superimposition. Two case studies with different phenomenon were included. Approximately six to sixteen coplanar (frontal) landmarks were selected to estimate the transformation and perspective projection parameters using a GA. Instead of considering coplanar landmarks, we strategically select potential noncoplanar landmarks in our proposed approach. We postulate that sole reliance on the landmarks in the front plane of the face to orient the skull without considering landmarks in the rear (ear) plane would lead to arbitrary tilting of the skull image to fit a face image and thus may lead to false positives. A true 

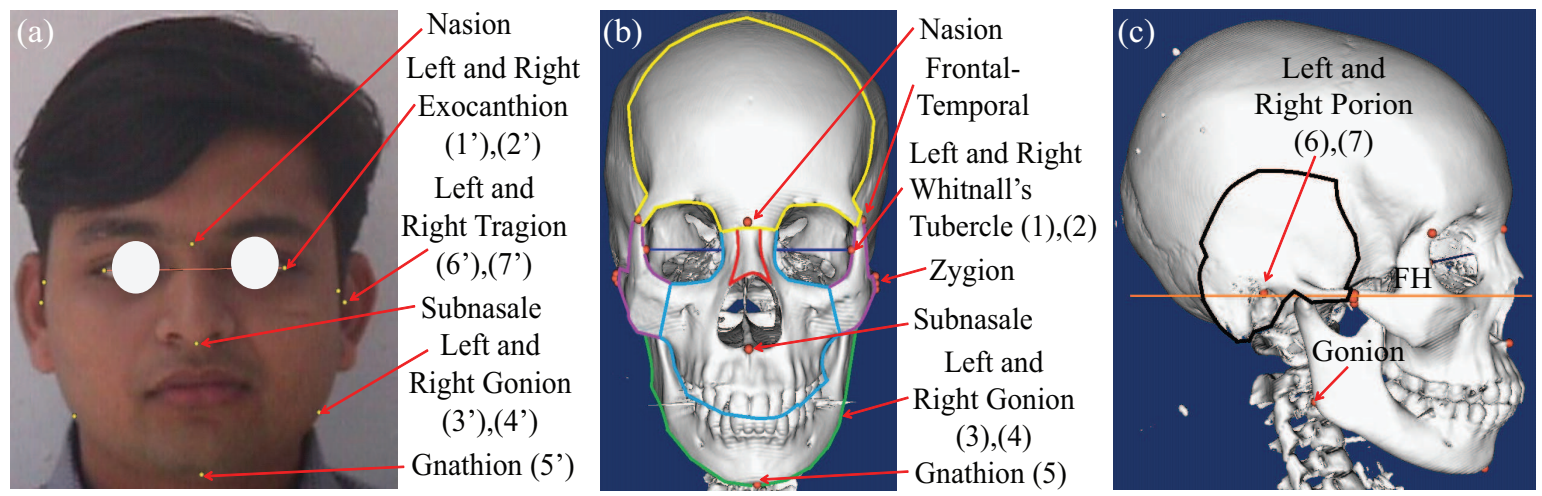

Fig. 1 Position of the landmarks on face and skull: (a) frontal view of a $2 \mathrm{D}$ face ${ }^{18}$, (b) frontal view of a 3D skull, and (c) side view of the 3D skull.

positive can only be determined when the skull is appropriately oriented to correspond to the posture in the face image.

For the proposed CAS, we initially select seven important anatomical landmarks from the frontal $(1,2,3,4,5)$ and rear $(6,7)$ plane of the digitized 3D skull model and 2D face images manually (Fig. 1). Then with the aid of the transformation parameters (scaling, rotation, and translation) the unknown skull is computed in the initialization stage and a given face is aligned. However, prior to the initialization, the $2 \mathrm{D}$ face points are reconstructed into $3 \mathrm{D}$ points by aligning them with an average face model to estimate the $z$-coordinate. Later, contours are generated using the cardinal spline at the mandible area of skulls and faces which would represent the characteristics of the targeted individual well. We then compute the local minima of the contours using the quasi-Newton method ${ }^{16}$ which would in turn serve as confidence parameters to classify the positive and false matches between the skull and the face images. Finally the true positive match is determined using the iterative closest point (ICP) algorithm ${ }^{17}$.

\section{CAS USING QUASI-NEWTON ICP}

Generally, appropriate subsampling of large sets of data points is an important pre-processing step that is usually carried out in many applications. When image sizes are large, they will substantially increase the computing overheads. Hence some intelligent strategies need to be incorporated in order to select potential adequate landmarks.

In the proposed CAS approach, only seven corresponding anatomical landmarks have been chosen. These landmarks are selected within their own acceptable area based on expert advice. The locations of these landmarks need to be significant enough to carry out the vital operations involved in the proposed approach. If the location of the landmarks selected are not close enough (within its area), this may lead to false inclusions. As shown in Fig. 1, typical anatomical landmarks (those that indicate bone muscles connection) include the left and right of Whitnall's tubercles $\left(\mathrm{Wt}^{\mathrm{L}}{ }_{(1)}\right.$ and $\left.\mathrm{Wt}^{\mathrm{R}}{ }_{(2)}\right)$, exocanthion $\left(\mathrm{ex}^{\mathrm{L}}{ }_{\left(1^{\prime}\right)}\right.$ and $\left.\mathrm{ex}_{\left(2^{\prime}\right)}^{\mathrm{R}}\right)$, the porions $\left(\mathrm{po}^{\mathrm{L}}{ }_{(6)}\right.$ and $\left.\mathrm{po}_{(7)}^{\mathrm{R}}\right)$ and tragions $\left(\operatorname{tr}_{\left(6^{\prime}\right)}^{\mathrm{L}}\right.$ and $\left.\operatorname{tr}^{\mathrm{R}}{ }_{\left(7^{\prime}\right)}\right)$. General cranial and facial landmarks for defining the contour include gonions $\left(\mathrm{go}_{K(3)}^{\mathrm{L}}, \mathrm{go}_{K(4)}^{\mathrm{R}}, \mathrm{go}_{F\left(3^{\prime}\right)}^{\mathrm{L}}\right.$ and $\left.\mathrm{go}_{F\left(4^{\prime}\right)}^{\mathrm{R}}\right)$ and gnathions $\left(\mathrm{gn}_{K(5)}\right.$ and $\left.\mathrm{gn}_{F\left(5^{\prime}\right)}\right)$. We note that Wt and po are the skull's landmarks which correspond to facial landmarks, ex and tr. They are located on the same horizontal line.

The proposed CAS approach comprises of three subprocesses:

(i) to align the unknown skull and a given face using an efficient initialization process;

(ii) to eliminate false matches using the quasiNewton method;

(iii) to ultimately determine the true positive match using R-ICP.

\section{Initialization process}

A good initial alignment would lead the algorithm to converge faster thereby yielding the global minimum at the final stage. At this stage, only four out of the seven landmarks from the frontal (Whitnall's tubercles, exocanthions) and rear plane (porions, tragions) from the skull and the face are selected for the transformation. It is sufficient to provide precise alignment using the basic geometrical transformations.

Let $F$ and $K$ represent the face and the skull 
anatomical landmarks, respectively. We can define them as

$$
\begin{aligned}
& F=\left\{\mathrm{ex}_{x}^{\mathrm{L}}, \mathrm{ex}_{y}^{\mathrm{L}}, \mathrm{ex}_{x}^{\mathrm{R}}, \mathrm{ex}_{y}^{\mathrm{R}}, \mathrm{tr}_{x}^{\mathrm{L}}, \mathrm{tr}_{y}^{\mathrm{L}}, \mathrm{tr}_{x}^{\mathrm{R}}, \mathrm{tr}_{y}^{\mathrm{R}}\right\} \\
& K=\left\{\mathrm{Wt}_{x}^{\mathrm{L}}, \mathrm{Wt}_{y}^{\mathrm{L}}, \mathrm{Wt}_{z}^{\mathrm{L}}, \mathrm{Wt}_{x}^{\mathrm{R}}, \mathrm{Wt}_{y}^{\mathrm{R}}, \mathrm{Wt}_{z}^{\mathrm{R}},\right. \\
& \left.\quad \mathrm{po}_{x}^{\mathrm{L}}, \mathrm{po}_{y}^{\mathrm{L}}, \mathrm{po}_{z}^{\mathrm{L}}, \mathrm{po}_{x}^{\mathrm{R}}, \mathrm{po}_{y}^{\mathrm{R}}, \mathrm{po}_{z}^{\mathrm{R}}\right\} .
\end{aligned}
$$

The 2D points of the face $\mathrm{ex}^{\mathrm{L}}, \mathrm{ex}^{\mathrm{R}}, \operatorname{tr}^{\mathrm{L}}$ and $\operatorname{tr}^{\mathrm{R}}$ are reconstructed to their respective 3D points in order to calculate all the rotation directions such as pitch, roll, and yaw. For the 2D points, the zcoordinate, which represents the angle of the rotation for the yaw direction, is not readily available. Hence the $z$-coordinate is computed using the 3D face models. First, a mean face model is required to estimate the $z$-coordinate of the $2 \mathrm{D}$ faces. It is generated by calculating the average position of all the selected points derived from the hundred available 3D face models ${ }^{19}$. In our case, instead of estimating the complete mean face model, only the four selected points of their mean positions are estimated, thereby reducing computational overheads. Procrustes analysis ${ }^{20}$ is used to normalize and align all the face models into a reference position and the mean position of the selected four points are calculated. Then the $2 \mathrm{D}$ points are reconstructed by aligning the $2 \mathrm{D}$ face landmarks with the mean points to estimate the $z$-coordinate. We assume that the $z$-coordinates of the $2 \mathrm{D}$ points are the same as the 3D corresponding points. By considering the $x$, $y$, and $z$ rotation coordinates, different corresponding points are selected to estimate the adjustment angles between the face and the skull. The 3D facial landmarks (previously assumed) are then updated with the estimated rotation parameters. With the updated facial landmarks, the rotation coordinates are estimated once again with the skull landmarks. This process is similar to translation and is carried out repeatedly until the parameters remain constant. Hence the 3D form of $F$ which has been reconstructed from its $2 \mathrm{D}$ form can be represented as

$$
\begin{aligned}
F=\left\{\mathrm{ex}_{x}^{\mathrm{L}}, \mathrm{ex}_{y}^{\mathrm{L}}, \mathrm{ex}_{z}^{\mathrm{L}}, \mathrm{ex}_{x}^{\mathrm{R}}, \mathrm{ex}_{y}^{\mathrm{R}}, \mathrm{ex}_{z}^{\mathrm{R}},\right. \\
\left.\quad \operatorname{tr}_{x}^{\mathrm{L}}, \operatorname{tr}_{y}^{\mathrm{L}}, \operatorname{tr}_{z}^{\mathrm{L}}, \operatorname{tr}_{x}^{\mathrm{R}}, \operatorname{tr}_{y}^{\mathrm{R}}, \operatorname{tr}_{z}^{\mathrm{R}}\right\} .
\end{aligned}
$$

Estimation of the relative life-size between the skull and the face using Wt and ex is discussed in Ref. 11. Hence, using the two relative landmarks $\mathrm{Wt}^{\mathrm{L}}, \mathrm{Wt}^{\mathrm{R}}$ of the skull and $\mathrm{ex}^{\mathrm{L}}, \mathrm{ex}^{\mathrm{R}}$ of the face, the scaling parameter $\mathbf{s}$ can be defined as

$$
\mathbf{s}=\frac{d_{\mathrm{ex}}}{d_{\mathrm{W}}} .
$$

Note that $d_{\text {ex }}$ is the distance between $\mathrm{ex}^{\mathrm{L}}$ and $\mathrm{ex}^{\mathrm{R}}$; $d_{\mathrm{Wt}}$ is the distance between $\mathrm{Wt}^{\mathrm{L}}$ and $\mathrm{Wt}^{\mathrm{R}}$. Assuming that our skull model has $n$ 3D points, the scaled model is computed using the scaling transformation

$$
\left[\begin{array}{c}
\tilde{x}_{i}^{\mathrm{K}} \\
\tilde{y}_{i}^{\mathrm{K}} \\
\tilde{z}_{i}^{\mathrm{K}}
\end{array}\right]=\mathbf{s}\left[\begin{array}{c}
x_{i}^{\mathrm{K}} \\
y_{i}^{\mathrm{K}} \\
z_{i}^{\mathrm{K}}
\end{array}\right]
$$

where $i=1, \ldots, n$. The $3 \mathrm{D}$ rotation matrix

$$
\begin{array}{r}
\mathbf{R}=\left(\begin{array}{ccc}
1 & 0 & 0 \\
0 & \cos \alpha & -\sin \alpha \\
0 & \sin \alpha & \cos \alpha
\end{array}\right)\left(\begin{array}{ccc}
\cos \beta & 0 & \sin \beta \\
0 & 1 & 0 \\
-\sin \beta & 0 & \cos \beta
\end{array}\right) \\
\times\left(\begin{array}{ccc}
\cos \gamma & -\sin \gamma & 0 \\
\sin \gamma & \cos \gamma & 0 \\
0 & 0 & 1
\end{array}\right)
\end{array}
$$

where $\alpha, \beta$, and $\gamma$ represent the differences in angles for each of the directions $x, y$, and $z$. These directions correspond, respectively, to pitch, roll, and yaw. The angle of the orientation for each of the directions is the difference in angle between the skull and the face which can be calculated on different planes. Each angle difference is estimated using different landmarks. The rotated skull model is given by

$$
\left[\begin{array}{c}
\bar{x}_{i}^{\mathrm{K}} \\
\bar{y}_{\dot{\mathrm{K}}}^{\mathrm{K}} \\
\bar{z}_{i}^{\mathrm{K}}
\end{array}\right]=\mathbf{R}\left[\begin{array}{c}
\tilde{x}_{i}^{\mathrm{K}} \\
\tilde{y}_{\dot{\mathrm{K}}}^{\mathrm{K}} \\
\tilde{z}_{i}^{\mathrm{K}}
\end{array}\right] .
$$

Then, the $\mathbf{T}$ parameter is computed using the distance between $\mathrm{ex}^{\mathrm{R}}$ and $\mathrm{Wt}^{\mathrm{R}}$

$$
\mathbf{T}=\left[\begin{array}{c}
T_{x} \\
T_{y} \\
T_{z}
\end{array}\right]=\left[\begin{array}{c}
\overline{e x}_{x}^{\mathrm{R}} \\
\overline{\mathrm{ex}} \overline{\mathrm{x}}_{y}^{\mathrm{R}} \\
\overline{\mathrm{ex}} \overline{\mathrm{K}}_{z}^{\mathrm{R}}
\end{array}\right]-\left[\begin{array}{c}
\overline{\mathrm{W}} \mathrm{t}_{x}^{\mathrm{R}} \\
\overline{\mathrm{W}} \mathrm{t}_{y}^{\mathrm{R}} \\
\overline{\mathrm{W}} \mathrm{t}_{z}^{\mathrm{R}}
\end{array}\right] .
$$

Finally, the skull is transformed completely using

$$
\left[\begin{array}{c}
\tilde{x}_{i}^{\mathrm{K}} \\
\tilde{y}_{\dot{\mathrm{K}}}^{\mathrm{K}} \\
\tilde{z}_{i}^{\mathrm{K}}
\end{array}\right]=\mathbf{s}\left[\begin{array}{c}
x_{i}^{\mathrm{K}} \\
y_{\dot{\mathrm{K}}}^{\mathrm{K}} \\
z_{i}^{\mathrm{K}}
\end{array}\right] \mathbf{R}+\mathbf{T} .
$$

The whole transformation procedure described above is intended to fit the skull into the face smoothly. It is an optimal process which is similar to the previous z-coordinate estimation process.

The 2D contour of the face on the jaw area is formed using $\mathrm{go}_{F}^{\mathrm{L}}, \mathrm{go}_{F}^{\mathrm{R}}$, and $\mathrm{gn}_{F}$ with the random pivot points that are in turn generated by the cardinal spline ${ }^{21}$. Pivot points are the points that are filled between the selected points along the 
curve. The contour of the transformed skull on the mandible area is also formed with the same method using $\mathrm{go}_{K}^{\mathrm{L}}, \mathrm{go}_{K}^{\mathrm{R}}$ and $\mathrm{gn}_{K}$, which only considers the $x$ and $y$ coordinates. The set of contours on the faces and skulls can be represented as

$$
\begin{aligned}
C^{\mathrm{F}} & =\left\{C^{F_{1}}, C^{F_{2}}, C^{F_{3}}, \ldots, C^{F_{m}}\right\} \\
C^{\mathrm{K}} & =\left\{C^{K_{1}}, C^{K_{2}}, C^{K_{3}}, \ldots, C^{K_{n}}\right\}
\end{aligned}
$$

where $m$ and $n$, respectively, represent the number of the faces and the number of skulls.

\section{Quasi-Newton method}

We incorporate the quasi-Newton method to eliminate false matches using the characteristics inherent on the contours. It has been used in image contour analysis ${ }^{22}$ using wavelet functions. In our approach, the contours can be modelled using Lagrange interpolating polynomial functions ${ }^{23}$. The Quasi-Newton method is used to determine the local minima of the functions and is based on Newton's method $^{24}$. In this method, the first and second order derivatives are determined to find the stationary points of the functions when the gradient is zero. The gradient and Hessian matrices are minimized where a Hessian matrix is a square matrix of second order partial derivatives which describes the local curvature of a function of multiple variables. In the quasi-Newton method, the Hessian matrix is obtained by analysing the successive gradient vectors with two important elements, namely, the search direction and step length for the gradient which avoids calculating derivatives.

Let the random pivot points generated by the cardinal spline of the $i$ th face and $j$ th skull be represented as

$$
\begin{aligned}
C^{F_{i}} & =\left\{x_{1}^{F_{i}}, y_{1}^{F_{i}}, x_{2}^{F_{i}}, y_{2}^{F_{i}}, \ldots, x_{p}^{F_{i}}, y_{p}^{F_{i}}\right\} \\
C^{K_{j}} & =\left\{x_{1}^{K_{j}}, y_{1}^{K_{j}}, x_{2}^{K_{j}}, y_{2}^{K_{j}}, \ldots, x_{p}^{K_{j}}, y_{p}^{K_{j}}\right\}
\end{aligned}
$$

where $p$ represents the number of contour points.

The polynomial functions of the contours are computed using the standard Lagrange's interpolation technique. It has been observed that a fourth degree polynomial is sufficient to construct the functions of these contours. The coefficients of this polynomial have been calculated using the points that are selected from the skull and the face. With the polynomial functions that represent the skull and the contour, the local minima of the functions can be computed using the Broyden-Fletcher-GoldfarbShanno (BFGS) algorithm ${ }^{25-28}$. The difference between the local minima of the skull and the face is calculated and adopted as the confidence parameter to eliminate false matches. By a suitable pre-defined threshold, the false matches are eliminated when the differences are high.

In Newton's method, the search direction is determined by the second order approximation of a function around $x_{j}$ :

$$
\begin{aligned}
f\left(x_{j}+\Delta x\right) \approx f\left(x_{j}\right) & +\Delta x^{\mathrm{T}} \nabla f\left(x_{j}\right) \\
& +\frac{1}{2} \Delta x^{\mathrm{T}} \nabla^{2} f\left(x_{j}\right) \Delta x .
\end{aligned}
$$

The approximation of the gradient for this function with respect to $\Delta x$ is

$$
\nabla f\left(x_{j}+\Delta x\right) \approx \nabla f\left(x_{j}\right)+\nabla^{2} f\left(x_{j}\right) \Delta x .
$$

By setting the gradient to zero we obtain,

$$
\Delta x=-Q_{j} \nabla f\left(x_{j}\right)
$$

where $Q_{j}=\left[\nabla^{2} f\left(x_{j}\right)\right]^{-1}$ is the inverse of the true Hessian matrix.

In quasi-Newton method, $Q_{j}$ is updated at each iteration using (16) which holds the properties of the inverse of the true Hessian matrix. BFGS is a robust method to update the approximate Hessian matrix, $Q_{j}$ where

$$
\begin{aligned}
Q_{j+1}=\left(I-V_{j} G_{j} y_{j}^{\mathrm{T}}\right) Q_{j}\left(I-V_{j} y_{j} G_{j}^{\mathrm{T}}\right) & \\
& +V_{j} G_{j} G_{j}^{\mathrm{T}}
\end{aligned}
$$

where $V_{j}=\left(y_{j}^{\mathrm{T}} G_{j}\right)^{-1}, y_{j}=\nabla f\left(x_{j+1}\right)-\nabla f\left(x_{j}\right), G_{j}=$ $x_{j+1}-x_{j}$, and $x_{j+1}=x_{j}+\phi_{j} \Delta x . \phi_{j}$ is determined using the line-search algorithm ${ }^{29}$ which searches for the descent direction along the interpolated function and further estimates the size of the step length.

The local minima of the contours of the face and the skull, $X_{i}^{\mathrm{F}}$ and $X_{i}^{\mathrm{K}}$ are obtained and these will be adopted as confidence parameters. $\left|X_{i}^{\mathrm{F}}-X_{i}^{\mathrm{K}}\right|$ is computed to get a measure of closeness of match between the face and the skull:

$$
M= \begin{cases}1, & \left|X_{i}^{\mathrm{F}}-X_{i}^{\mathrm{K}}\right|<1.0 \\ 0, & \text { otherwise }\end{cases}
$$

Eq. (18) is used to decide on retaining the possible set of positive matches $M$ from the dataset; otherwise it will be retained to be considered for the next stage which is the execution of the R-ICP algorithm. The threshold value in (18) is decided based on the analysis carried out based on the experiments. Logically, the matching pair with closed characteristics will always obtain low absolute differences. 


\section{Rotational iterative closest point (R-ICP)}

ICP is a point-based technique which has been applied in various fields especially in image and shape registrations ${ }^{30,31}$. We employ the R-ICP algorithm to register the pivot points between the skull and the face. As there are not more than 100 pivot points, the conventional ICP algorithm ${ }^{17}$ is sufficient to perform the registration process. The algorithm determines the correspondence between the closest target and model points using the distance. The errors of the correspondences are measured based on the mean squared error (MSE) cost function which are represented in terms of pixels. In our case, rotational ICP is applied to perform the rigid registration between the face and the skull point sets. The registration needs to be rigid so that the contours between the face and the skull do not deform. To achieve a reasonable alignment, only the rotation operation is considered and translation is ignored. This ensures that the horizontal line between ex and Wt remains static.

Let the possible positive matches yielded by the quasi-Newton method for a given skull and the set of available faces be represented by $\left\{C^{\mathrm{K}}\right\}$ and $\left\{C^{F_{1}}, C^{F_{2}}, C^{F_{3}}, \ldots, C^{F_{m}}\right\}$ where $m$ is the number of faces. These are the confidence candidates (face contours) which correspond to the skull. The correspondence between the pivot points of the given skull and any $l$ th face is determined based on the nearest point yielded by the distance given by

$$
\begin{aligned}
& d\left(C^{\mathrm{K}}, C^{F_{l}}\right) \\
& =\sum_{i=1}^{p} \sqrt{\left(x_{i}^{\mathrm{K}}-x_{i}^{F_{l}}\right)^{2}+\left(y_{i}^{\mathrm{K}}-y_{i}^{F_{l}}\right)^{2}} .
\end{aligned}
$$

All the contour points of the skull model can be aligned by applying the following rotation transformation

$$
\left[\begin{array}{l}
\hat{C}_{x_{i}}^{\mathrm{K}} \\
\hat{C}_{y_{i}}^{\mathrm{K}}
\end{array}\right]=\mathbf{R}\left[\begin{array}{l}
C_{x_{i}}^{\mathrm{K}} \\
C_{y_{i}}^{\mathrm{K}}
\end{array}\right]
$$

where $i=1, \ldots, p$ and $\left(\hat{x}_{i}^{\mathrm{K}}, \hat{y}_{i}^{\mathrm{K}}\right)$ represents the transformed points.

Error estimates based on the MSE for the transformed skull points can be computed using

$$
e=\sum_{i=1}^{p} \sqrt{\left(\hat{x}_{i}^{\mathrm{K}}-x_{i}^{F_{m}}\right)^{2}+\left(\hat{y}_{i}^{\mathrm{K}}-y_{i}^{F_{m}}\right)^{2}} .
$$

The R-ICP algorithm is iterated and a stopping criterion is set such that if the error remains constant for a certain number of iterations, the algorithm will
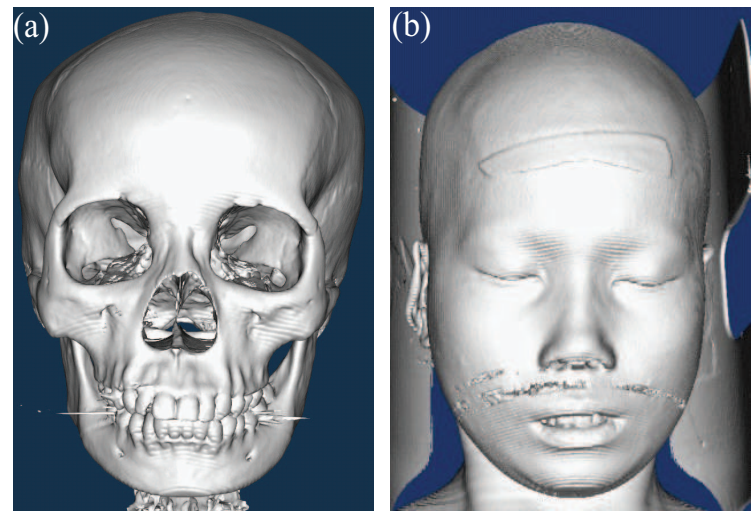

Fig. 2 Example of the female skull model (3D-CT scan data) and the associated facial visualizations: (a) extracted female skull; (b) extracted female face (S2).

terminate.

$$
F= \begin{cases}1, & e_{i}<e_{i-1}, \\ 0, & \text { otherwise. }\end{cases}
$$

The matching pair which yields the least error will be decided as the final match, $F$, using (22).

\section{MATERIALS (EXTRACTED 3D SKULLS AND 2D FACE PHOTOS)}

For experimental evaluations, we use the scanned 3D data of two human heads which have been acquired from 3D-CT (computed tomography) scans. The soft features of the face and the features of the skulls (Fig. 2) were extracted from the CT data using the visualization toolkit (VTK) ${ }^{32}$, which has been commonly used in medical imaging. The skulls and facial soft tissues were extracted using the marching cubes computer graphics algorithm ${ }^{33}$.

Fig. 2 shows an instance of typical visualizations of the female 3D-CT scan data. Let S1 and S2 represent the $2 \mathrm{D}$ face images of the male subject and the female subject, respectively, which basically represent their facial soft features that are extracted from the 3D-CT scan data. These two face images represent the related face images for the skulls that can lead to positive matches during the experiments carried out in this study. The 2D face images are manually captured using the screenshot utility tool that is available in VTK. Facial landmarks were selected directly from these 2D images instead of extracting from the 3D data. From Fig. 2b, we can see that it closely resembles the appearance of the actual face of the subject. Also, apart from its face texture, facial features such as nose, mouth, and jaw are clearly visualized with the aid of VTK. In craniofacial superimposition, the matching criteria is usu- 
ally emphasized more on the facial features rather than the face textures. Since the available 3D-CT scan data that represent the skulls and their positive matches (face images) are from Asian subjects, we consider acquiring the other face images also from Asian subjects for our experimental evaluations. To represent the exclusions (negative matches), i.e., those face images that do not correspond to the $3 \mathrm{D}$ skulls used in the experiments, we use 27 face images comprising of 12 females and 15 males. These 2D face images have been acquired from various sources including a public face database ${ }^{18}$. Most of the face images used in our experiments are near frontal (frontal and slightly oblique) without facial expressions and intended for identification purposes.

The experiments include 58 craniofacial superimpositions that have been carried out using the two 3D skulls and twenty-nine 2D face images. The male skull is superimposed with its related 2D face image (S1) as well as with the other face images. The female skull is also superimposed with its related 2D face image (S2) as well as other images.

\section{RESULTS AND DISCUSSION}

The main purpose of our experimental evaluation is to investigate the robustness of the proposed approach in order to determine the final (true) positive match out of all the superimpositions. In other words, we intend to find the best matching pair between the unknown skulls and the given set of faces. Hence the following two important aspects of the proposed approach are evaluated:

(i) Matching with or without initialization will be, respectively, referred to as case 1 and case 2 .

(ii) The robustness of the quasi-Newton method and R-ICP algorithms pertaining to the proposed craniofacial superimposition approach.

We first find the significance of the initial alignment (initialization) in the approach. Then the robustness of the quasi-Newton method and R-ICP to determine the best matching pair are examined.

\section{Impact of initialization over R-ICP}

The purpose of this experiment is to compare the approach with and without the initialization process using the R-ICP algorithm. We recall that the objective of the R-ICP algorithm is to determine the true positive match. The correspondences between the contour points of the unknown skull and the given set of faces have been measured based on the standard MSE cost function. The matching pair that

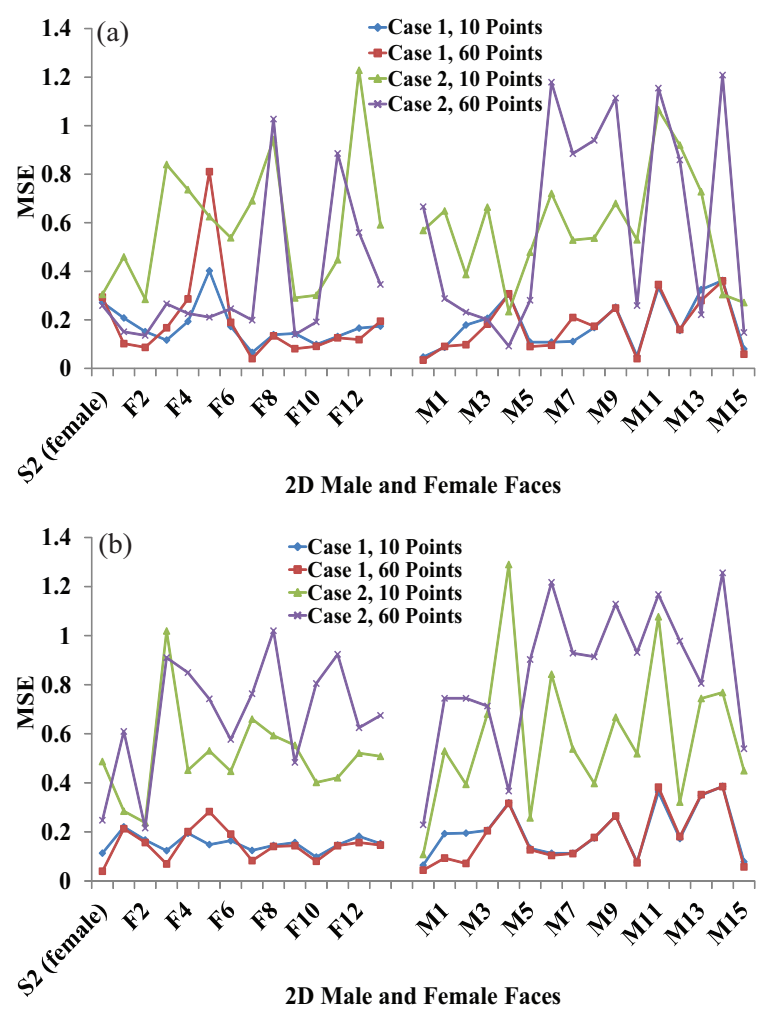

Fig. 3 MSE response for the two cases when tested with the superimposition of $3 \mathrm{D}$ skulls and the $2 \mathrm{D}$ faces: (a) superimposition of 3D male skull and all the faces; (b) superimposition of 3D female skull and all the faces.

yields the least MSE among the data is taken as the true positive match.

Fig. 3 and Table 1 show the MSE response for the superimpositions carried out for cases 1 and 2 with respect to S1 and S2. From Fig. 3, it can be observed that the initialization process plays an important role in finding the true positive match. We observe that without the initialization process, the R-ICP algorithm was unable to find the positive match for the male and female skulls by making use of MSE estimates. In initialization, amounts of pivot points that filled between the selected points along the curves are also considered. As shown in Fig. 3, 60 pivot points give good results as it produces the lowest MSE.

To determine the significance of the MSE between the two cases, we used a $t$-test. The MSE of case 1 (refer to Table 1 ) has been compared with the MSE of case 2 using the chosen two sets of pivot points of the two skulls. We conclude that the two cases differ significantly pertaining to the matching performed by the R-ICP algorithm. 
Table 1 MSE response for the two cases when tested with the superimposition of skulls and all the faces.

\begin{tabular}{|c|c|c|c|c|c|c|c|c|}
\hline \multirow{3}{*}{$\begin{array}{l}2 \mathrm{D} \\
\text { faces }\end{array}$} & \multicolumn{4}{|c|}{ 3D male skull } & \multicolumn{4}{|c|}{ 3D female skull } \\
\hline & \multicolumn{2}{|c|}{10 pivot points (P1) } & \multicolumn{2}{|c|}{60 pivot points (P2) } & \multicolumn{2}{|c|}{10 pivot points (P3) } & \multicolumn{2}{|c|}{60 pivot points (P4) } \\
\hline & case 1 & case 2 & case 1 & case 2 & case 1 & case 2 & case 1 & case 2 \\
\hline S1 & 0.0471 & 0.569 & 0.0345 & 0.666 & 0.0647 & 0.108 & 0.0442 & 0.229 \\
\hline S2 & 0.272 & 0.305 & 0.294 & 0.259 & 0.113 & 0.487 & 0.0399 & 0.248 \\
\hline F1 & 0.208 & 0.459 & 0.102 & 0.151 & 0.221 & 0.285 & 0.213 & 0.610 \\
\hline $\mathrm{F} 2$ & 0.151 & 0.285 & 0.0868 & 0.136 & 0.168 & 0.238 & 0.157 & 0.216 \\
\hline F3 & 0.117 & 0.840 & 0.167 & 0.266 & 0.123 & 1.019 & 0.0696 & 0.910 \\
\hline F4 & 0.194 & 0.736 & 0.286 & 0.226 & 0.195 & 0.452 & 0.201 & 0.849 \\
\hline F5 & 0.402 & 0.625 & 0.810 & 0.211 & 0.148 & 0.531 & 0.283 & 0.742 \\
\hline F6 & 0.173 & 0.538 & 0.190 & 0.245 & 0.164 & 0.448 & 0.191 & 0.577 \\
\hline F7 & 0.0655 & 0.691 & 0.0404 & 0.200 & 0.125 & 0.660 & 0.0829 & 0.763 \\
\hline F8 & 0.139 & 0.943 & 0.134 & 1.027 & 0.150 & 0.593 & 0.141 & 1.020 \\
\hline F9 & 0.144 & 0.290 & 0.0810 & 0.139 & 0.157 & 0.553 & 0.144 & 0.484 \\
\hline F10 & 0.0986 & 0.301 & 0.0909 & 0.191 & 0.0975 & 0.402 & 0.0803 & 0.805 \\
\hline F11 & 0.132 & 0.447 & 0.126 & 0.885 & 0.147 & 0.421 & 0.144 & 0.924 \\
\hline F12 & 0.166 & 1.228 & 0.119 & 0.559 & 0.182 & 0.521 & 0.157 & 0.625 \\
\hline M1 & 0.0869 & 0.649 & 0.0906 & 0.288 & 0.193 & 0.530 & 0.0935 & 0.745 \\
\hline M2 & 0.179 & 0.387 & 0.098 & 0.232 & 0.195 & 0.394 & 0.0715 & 0.745 \\
\hline M3 & 0.206 & 0.665 & 0.183 & 0.201 & 0.207 & 0.680 & 0.205 & 0.713 \\
\hline M4 & 0.306 & 0.234 & 0.307 & 0.0921 & 0.319 & 1.290 & 0.316 & 0.367 \\
\hline M5 & 0.108 & 0.479 & 0.090 & 0.281 & 0.133 & 0.257 & 0.127 & 0.903 \\
\hline M6 & 0.108 & 0.721 & 0.0955 & 1.179 & 0.113 & 0.843 & 0.104 & 1.217 \\
\hline M7 & 0.111 & 0.529 & 0.210 & 0.885 & 0.113 & 0.5338 & 0.118 & 0.929 \\
\hline M8 & 0.168 & 0.537 & 0.174 & 0.940 & 0.175 & 0.398 & 0.178 & 0.914 \\
\hline M9 & 0.252 & 0.680 & 0.249 & 1.114 & 0.266 & 0.668 & 0.265 & 1.129 \\
\hline M10 & 0.0508 & 0.530 & 0.0404 & 0.259 & 0.077 & 0.519 & 0.0744 & 0.931 \\
\hline M11 & 0.334 & 1.066 & 0.346 & 1.155 & 0.363 & 1.077 & 0.382 & 1.167 \\
\hline M12 & 0.156 & 0.920 & 0.160 & 0.859 & 0.173 & 0.322 & 0.181 & 0.978 \\
\hline M13 & 0.325 & 0.728 & 0.280 & 0.221 & 0.349 & 0.744 & 0.352 & 0.805 \\
\hline M14 & 0.362 & 0.304 & 0.360 & 1.208 & 0.386 & 0.769 & 0.385 & 1.256 \\
\hline M15 & 0.0803 & 0.271 & 0.0586 & 0.148 & 0.0786 & 0.450 & 0.0573 & 0.539 \\
\hline
\end{tabular}

As can be seen in Fig. 4a and Fig. 4b, the proposed approach has yielded well superimposed positive matches using the four frontal and rear landmarks. We also observe that the ex and $\mathrm{Wt}$ have been well aligned horizontally as depicted in the figure. Similarly $\operatorname{tr}^{\mathrm{L}}$ and $\operatorname{tr}^{\mathrm{R}}$ have also been well fitted with $\mathrm{po}^{\mathrm{L}}$ and $\mathrm{po}^{\mathrm{R}}$ between the face and the skull. In Fig. 4c and Fig. 4d, the face and the skull have not been well aligned with each other. The direction of the skulls are facing away from the face and all of the landmarks are not well fitted with each other.

In summary, the above experimental results show that the initialization stage is important to improve the performance of matching. This justifies the fact that the transformation parameters for lifesize and orientation adjustment can be estimated precisely with the suggested method. When the number of pivot points of the contours is set to
60, the R-ICP algorithm is able to provide precise outputs for all the cases of the superimpositions that have been considered.

\section{Results of experiments with the quasi-Newton method}

The purpose of this experiment is to test the feasibility of the quasi-Newton method which form part of the proposed CAS approach. We test the feasibility of the quasi-Newton method on determining positive and false matches. Fig. 5 shows the absolute difference (refer to (18)) between the 3D female skull and the 2D faces. If the absolute difference of the true positive for both the skulls is lower than 1.0, then it indicates a potential positive match. Further, we notice that the absolute difference for both the skulls are not affected by different numbers of pivot points (Fig. 5a,b).

The quasi-Newton method could successfully 

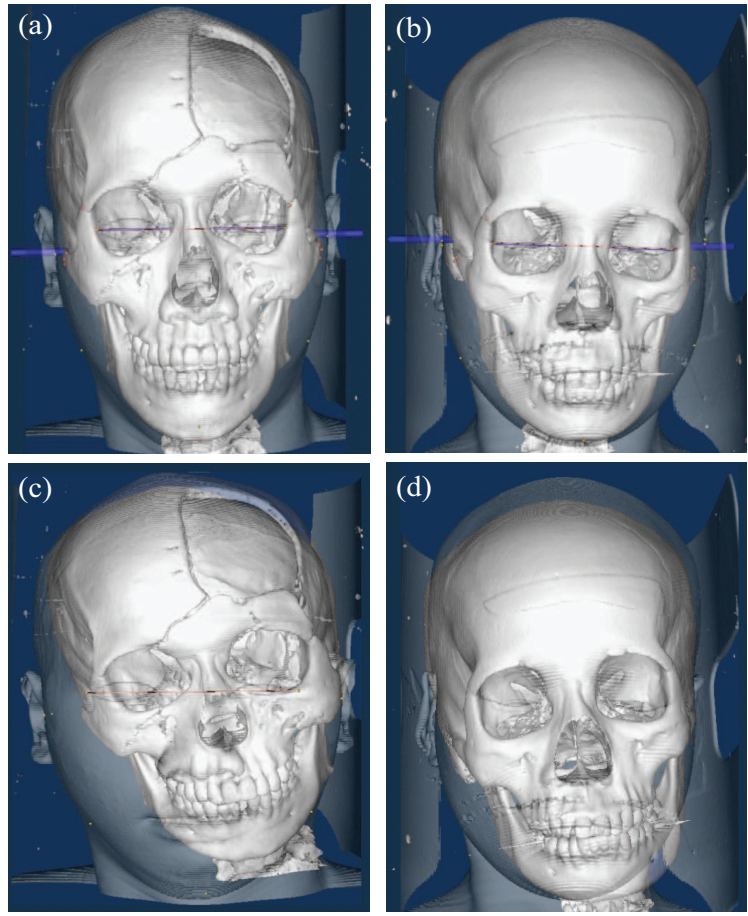

Fig. 4 Final outputs of superimpositions of related skull and face images (true positives) yielded by the R-ICP algorithms of the proposed CAS approach: (a) S1, true positive case of the male skull with initialization; (b) S2, true positive case of the female skull with initialization; (c) S1, true positive case of the male skull without initialization; (d) S2, true positive case of the female skull without initialization.

eliminate 22 and 19 out of the 29 false matches, respectively, for the male and the female skull superimpositions. Fig. 6 shows two typical outputs that have yielded high and low absolute differences. As shown in Fig. 6a, the outline of the female skull mandible has crossed the outline of the subject's (female 7) jaw area where the contours did not fit well with each other. Consequently, a high absolute difference has been produced as the characteristics between the skull contour and the face contour significantly varied from each other whereby in Fig. 6b, the mandible outline of the skull has been fitted along the subject's (male 5) jaw area. This has resulted in yielding a low absolute difference as the characteristics between the skull contour and the face contour are quite close to each other.

In summary, the quasi-Newton method employed in the proposed CAS is able to retain the true positive matches which are the inputs to the subsequent R-ICP algorithm. Furthermore, its per-
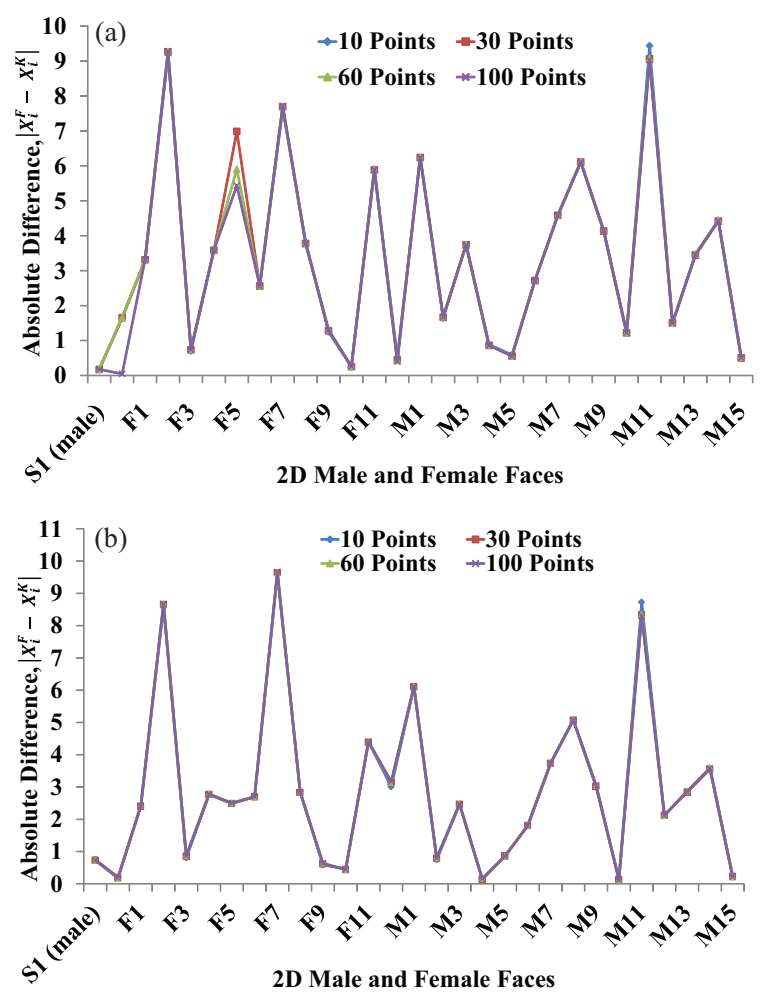

Fig. 5 Key results of the quasi-Newton method of the proposed CAS approach: (a) absolute difference yielded by the quasi-Newton method when a 3D male skull was imposed with the available 2D faces; (b) absolute difference yielded by the quasi-Newton method when a 3D female skull was imposed with the available 2D faces.

formance is not affected by the number of pivot points that are assigned.

\section{CONCLUSIONS}

Since 1991, all procedures described for computerizing the CS indicate that the matching has been obtained relying on the landmarks in the front (eye) plane without mentioning the use of life-size images. This study, for the first time, proposes a new CAS method to orient the skull image, taking into consideration the relationship between Whitnall's tubercles and the porions in the skull and the exocanthions and the tragus in the face image which are maintained in the life-size. For this purpose, we have used a quasi-Newton ICP approach and the validity of achieving an appropriate orientation have been illustrated using skull and the face images that are related as well as unrelated. Based on the results, the quasi-Newton method proposed is able to preserve the ground truth in positive as well as negative matches. The R-ICP algorithm is able 

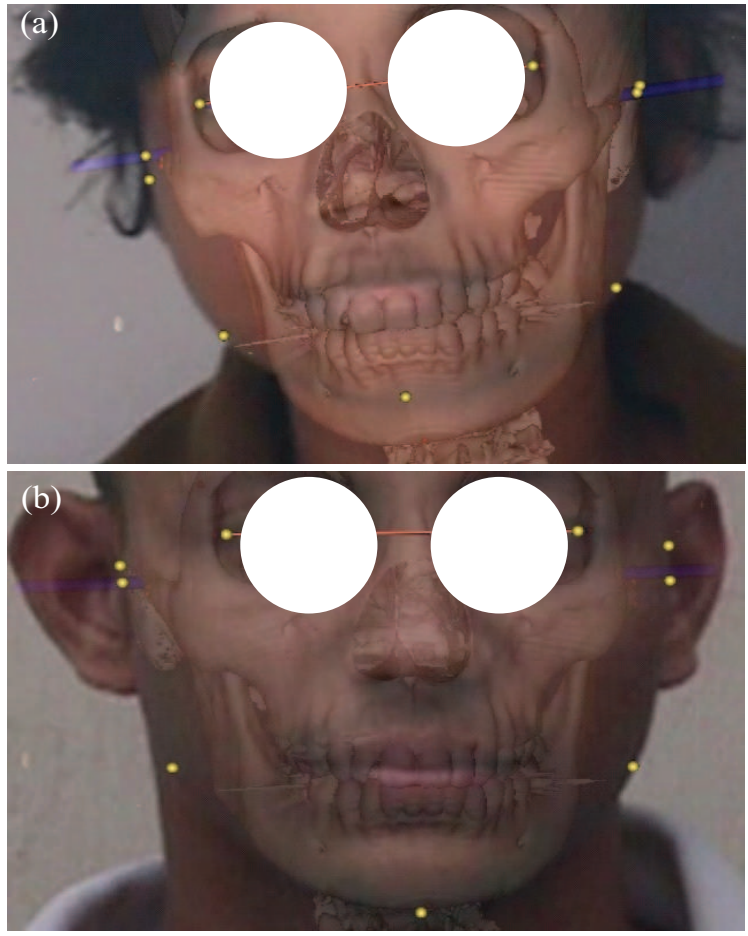

Fig. 6 Typical outputs yielded by the quasi-Newton method after the initialization process of the proposed CAS approach: (a) typical false match (female skull being superimposed with F7); (b) typical false positive match (female skull being superimposed with M5).

to provide precise outputs for all the cases of the superimpositions that have been considered. The efficiency and validity of the approach demonstrated will help increasing the confidence level of experts during the decision making process of craniofacial superimposition.

Acknowledgements: This study is supported by the RUPRGS Universiti Sains Malaysia (1001/PKOMP/846051) and RUI grant (1001/PKOMP/811290). We would like to thank Bahari Belaton and K.G. Subramaniam for providing valuable comments to improve this paper.

\section{REFERENCES}

1. Ricci A, Marella GL, Apostol MA (2006) A new experimental approach to computer-aided face/skull identification in forensic anthropology. Am J Forensic Med Pathol 27, 46-9.

2. Gordon GM, Steyn M (2012) An investigation into the accuracy and reliability of skull-photo superimposition in a South African sample. Forensic Sci Int 216, 198.e1-6.

3. Nickerson BA, Fitzhorn PA, Koch SK, Charney M (1991) A methodology for near-optimal computa- tional superimposition of two-dimensional digital facial photographs and three-dimensional cranial surface meshes. $J$ Forensic Sci 36, 480-500.

4. Ibáñez O, Cordón O, Damas S, Santamaría J (2008) Craniofacial superimposition based on genetic algorithms and fuzzy location of cephalometric landmarks. In: Hybrid Artificial Intelligence Systems, Springer, Berlin, pp 599-607.

5. Ballerini L, Cordón O, Santamaría J, Damas S, Alemán I, Botella M (2007) Craniofacial superimposition in forensic identification using genetic algorithms. In: Proceedings of the 3rd International Symposium on Information Assurance and Security, IEEE Computer Society, pp 429-34.

6. Ibáñez O, Cordón O, Damas S, Santamaría J (2009) Multimodal genetic algorithms for craniofacial superimposition. In: Chiong R (ed) Nature Inspired Informatics for Intelligent Applications and Knowledge Discovery Implications in Business Science and Engineering, IGI Global, pp 119-42.

7. Santamaría J, Cordón O, Damas S, Ibáñez O (2009) 3D-2D image registration for craniofacial superimposition in forensic medicine using covariance matrix adaptation evolution strategy. In: 9th International Conference on Information Technology and Applications in Biomedicine, pp 1-4.

8. Ibáñez O, Cordón O, Damas S (2012) A cooperative coevolutionary approach dealing with the skull-face overlay uncertainty in forensic identification by craniofacial superimposition. Soft Comput 16, 797-808.

9. Campomanes-Álvarez BR, Ibáñez O, Navarro F, Alemán I, Botella M, Damas S, Cordón O (2014) Computer vision and soft computing for automatic skull-face overlay in craniofacial superimposition. Forensic Sci Int 245, 77-86.

10. Campomanes-Álvarez BR, Ibáñez O, CampomanesÁlvarez C, Damas S, Cordón O (2015) Modeling facial soft tissue thickness for automatic skull-face overlay. IEEE Trans Inform Foren Secur 10, 2057-70.

11. Jayaprakash PT, Srinivasan GJ, Amravaneswaran MG (2001) Cranio-facial morphanalysis: a new method for enhancing reliability while identifying skulls by photo superimposition. Forensic Sci Int 117, 121-43.

12. Sekharan PC (1989) The problems of positioning skulls for video superimposition technique. Can Soc Forensic Sci J 22, 21-5.

13. Maat GJR (1989) The positioning and magnification of faces and skulls for photographic superimposition. Forensic Sci Int 41, 225-35.

14. Ghosh AK, Sinha P (2001) An economised craniofacial identification system. Forensic Sci Int 117, 109-19.

15. Santamaría J, Cordón O, Damas S, Ibáñez O (2009) Tackling the coplanarity problem in 3D camera calibration by means of fuzzy landmarks: a performance study in forensic craniofacial superimposition. In: IEEE 12th International Conference on Computer Vi- 
sion Workshops, IEEE Computer Society, pp 1686-93.

16. Davidon WC (1991) Variable metric method for minimization. SIAM J Optim 1, 1-17.

17. Besl PJ, McKay ND (1992) A method for registration of 3-D shapes. IEEE Trans Pattern Anal Mach Intell 14, 239-56.

18. Jain V, Mukherjee A (2002) The Indian face database, vis-www.cs.umass.edu/ vidit/indianfacedatabase/.

19. Blanz V, Vetter $T$ (1999) A morphable model for the synthesis of 3D faces. In: Proceedings of the 26th Annual Conference on Computer Graphics and Interactive Techniques, pp 187-94.

20. Kendall DG (1989) A survey of the statistical theory of shape. Stat Sci 4, 87-99.

21. Catmull E, Rom R (1974) A class of local interpolating splines. Computer Aided Geometric Design, Academic Press, pp 317-26.

22. Antoshchuk S, Nikolenko A, Babilunga O, Kapunova $\mathrm{K}$ (2009) Image contour analysis using iterative search algorithms based on wavelet transform. In: IEEE International Workshop on Intelligent Data Acquisition and Advanced Computing Systems: Technology and Applications, pp 390-3.

23. Meijering E (2002) A chronology of interpolation: From ancient astronomy to modern signal and image processing. In: Proceedings of the IEEE, pp 319-42.

24. Atkinson KE (2008) An Introduction to Numerical Analysis, 2nd edn, Wiley.

25. Broyden CG (1967) Quasi-Newton methods and their application to function minimisation. Math Comput 21, 368-81.

26. Fletcher R (1970) A new approach to variable metric algorithms. Comput J 13, 317-22.

27. Goldfarb D (1970) A family of variable-metric methods derived by variational means. Math Comput 24 23-6.

28. Shanno DF (1970) Conditioning of quasi-Newton methods for function minimization. Math Comput 24, 647-56.

29. Box MJ, Davies D, Swann WH, Industries IC (1981) Non-linear Optimization Techniques, Institute of Manpower Studies, Brighton.

30. Liu Y, Martin RR, De Dominicis L, Li B (2014) Using Retinex for point selection in 3D shape registration. Pattern Recogn 47, 2126-42.

31. Lu X, Jain AK (2005) Deformation analysis for 3D face matching. In: 7th IEEE Workshop on Application of Computer Vision, IEEE Computer Society, vol 1, pp 99-104.

32. Schroeder WJ (1998) The Visualization Toolkit User's Guide: Updated for Version 4.0, Kitware.

33. Lorensen WE, Cline HE (1987) Marching cubes: A high resolution 3D surface construction algorithm. ACM SIGGRAPH Comput Graph 21, 163-9. 